\title{
Metabolomics: Approaches and Applications to Diabetes Research
}

\author{
Anju Preet ${ }^{1}$, Tejaswita M. Karve ${ }^{2}$, Nasser Rizk ${ }^{3}$ and Amrita K. Cheema ${ }^{1 *}$
}

${ }^{1}$ Department of Oncology, Lombardi Comprehensive Cancer Center, Georgetown University School of Medicine, Washington, USA

${ }^{2}$ Department of Biochemistry and Cellular and Molecular Biology, Lombardi Comprehensive Cancer Center, Georgetown University School of Medicine, Washington, USA ${ }^{3}$ Department of Health Sciences, Qatar University, Doha, Qata

\begin{abstract}
Diabetes mellitus is a multi-factorial disease associated with a dysregulated metabolism. A holistic approach emphasizing each of the individual factors with regards to complete pathophysiology of the disease is critical to our understanding of this heterogeneous disease. Several technical advances in the field of functional genomics such as metabolomics and proteomics aid in comprehending the state of the overall biological system and thus can be utilized to decipher the complex interactions among components of the metabolic system in human diabetes. Above-mentioned techniques combined with a set of bioinformatics tools and available databases aim to profile wide array of proteins and metabolites repertoire in humans. The accurate and comprehensive measurements of these molecules is employed to investigate complex interactions of metabolites and proteins not only among themselves but also with genes, transcripts and other small molecules to decipher cellular microenvironment and the effects due to drug intervention. This review provides an overview of the applications of metabolomics in human diabetes research. We also discuss the potentia of combining different bioinformatics tools with the omics approach to advance the scientific knowledge towards discovery of biomarkers to monitor and regulate general human health with respect to the deregulated metabolic state, a characteristic of diabetes mellitus.
\end{abstract}

Keywords: Diabetes; Metabolomics; Gas chromatography; Liquid chromatography; Non-magnetic resonance

\section{Introduction}

Diabetes mellitus is a chronic metabolic disease, characterized by elevated blood glucose level-hyperglycemia, due to a deficient insulin secretion by pancreas or an inefficient insulin action in body tissues $[1,2]$. World Health Organization (WHO) estimated 3.4 million diabetes related deaths in 2004 and projected the incidence of diabetes to double between 2005 and 2030 [3,4]. Four types of diabetes mellitus have been identified: Type1, Type 2, "other specific types" and gestational diabetes [5]. Type 2 Diabetes (T2D) is the most prevalent form of diabetes characterized by decreased insulin sensitivity as opposed to deficient insulin secretion due to autoimmune destruction of pancreatic betacells, the accepted causal factor for T1D [2,6]. Persistent hyperglycemia in diabetes leads to metabolic dysfunction and manifests in form of series of complications like retinopathy, neuropathy, nephropathy and cardiovascular disease [7]. Extensive research efforts have been invested in order to understand the metabolic signature of T2D that would augment early detection of the disease and the development of effective therapeutics [8]. Metabolite analysis in body fluids such as blood and urine is routinely practiced to assess diabetes risk $[9,10]$. Metabolomics is a powerful tool to study the complexities of T2D development and progression. Metabolic phenotype is a reflection of genetic makeup that reflects the changes induced by cellular and external environmental conditions that manifest in the form of diseases of altered metabolism such as T2D. Better understanding of metabolic status in T2D would aid clinical interventions to direct the metabolism in more favorable direction. This review summarizes the applications of metabolomics in diabetes research.

\section{Metabolomics as a Tool for Diabetes Research}

Metabolomics is defined as a comprehensive characterization of endogenous metabolites representing the "metabolome". It provides global analysis of small molecules, which are either substrates or are products of metabolism.

Metabolomics investigates the unique metabolic phenotype or fingerprint that provides a snapshot of all metabolic pathways in an organism at any given time. It is emerging as an important tool for the study of diseases of dysregulated systemic metabolism such as obesity, cardiovascular disease, diabetes mellitus and associated complications, as it focuses on identifying biochemical pathways and their interactive roles within systemic metabolism [11,12]. Metabolome, the quantitative complement of metabolites in a biological system, is traditionally placed at the lowest tier of the biochemical information flow, originating from genome transcriptome that translates to proteome to metabolome (Figure 1)

Metabolome is considered a sensitive indicator of both genetic and environmental perturbations. Metabolic reconstructions suggest that the changes in the metabolome are usually greater than those observed at protein or gene level $[13,14]$. Each level of a biological system interacts with each other and elicits a characteristic response to intrinsic as well as intrinsic environmental challenges (diet, lifestyle, drug and disease) to determine the resultant phenotype. Metabolomics can be a top down study of a biological system, using a holistic approach involving the study of components and interactions of the complete system. Bottoms-up strategy on the other hand, refers to the study of specific components and interactions within the system. The study of metabolites provides insights into biological processes, facilitating the understanding and manipulation of complex biological systems for diagnostic, prognostic and therapeutic purposes.

Corresponding author: Dr. Amrita K. Cheema, Department of Oncology, Lombardi Comprehensive Cancer Center, Georgetown University, 3900 Reservoir Road, NW, Washington D.C. 20057, USA, Tel: 202-687-2756; Fax: 202-687-8860 E-mail: akc27@georgetown.edu

Received December 02, 2011; Accepted January 14, 2012; Published January 14,2012

Citation: Preet A, Karve TM, Rizk N, Cheema AK (2012) Metabolomics: Approaches and Applications to Diabetes Research. J Diabetes Metab S6:001. doi:10.4172/2155-6156.S6-001

Copyright: ( $) 2012$ Preet A, et al. This is an open-access article distributed unde the terms of the Creative Commons Attribution License, which permits unrestricted use, distribution, and reproduction in any medium, provided the original author and source are credited. 


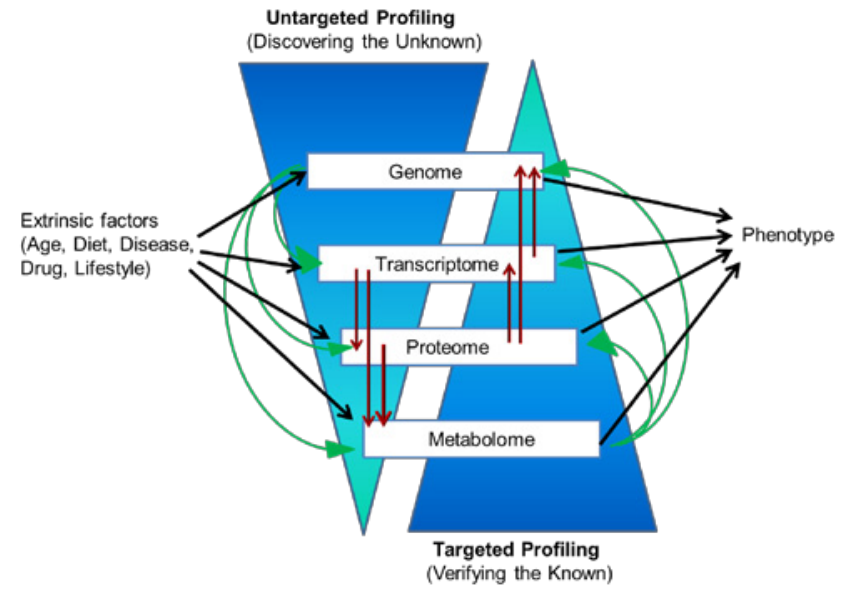

Figure 1: Deciphering the outcome of complex interplay between extrinsic and intrinsic factors in biological systems using metabolomics. Different functional levels in a biological system such as genome, transcriptome, proteome and metabolome interact with each other via complex flow of bi-directional information. Each level is influenced by environmental factors such as diet, drug, disease, lifestyle and age, which in turn dictate the phenotype of the biological system. Metabolomics uses untargeted profiling to discover alterations in metabolome arising out of complex interactions. A targeted profiling approach is instrumental in verifying alterations known to exist due to complex interplay of different factors thus providing tools for hypothesis testing in a diabetes research.

Two major approaches are currently used in metabolomics: targeted and untargeted. Targeted studies focus on quantitative measurements of specific metabolites with high precision. These studies involve the use of biochemical and analytical tools for the quantification of known metabolites of biological interest. Targeted studies assist the investigator with hypothesis testing and require the addition/spiking of several stable isotope-labeled standards as appropriate internal standards to ensure accuracy and specificity of quantitation. Targeted, quantitative metabolomics have facilitated the characterization of known as well as novel metabolic changes in experimental diabetic mice as well as diabetes patients [15-17]. Recent studies using a targeted metabolomics approach revealed altered and modified metabolic phenotype in diabetes and drug treatment respectively $[16,18,19]$. Analysis of dysregulated metabolites associated with diabetes, provide a functional readout of the metabolic state of subject under study and helps identification of candidate markers of metabolic pathways affected by the disease and/or treatment. Pathway specific perturbations of metabolic homeostasis in individuals can help identify patients at high risk and can predict diagnosis and prognosis of the diseased state $[18,20]$.

Non-targeted metabolomic profiling on the other hand, does not require prior knowledge and can thus be used to identify novel metabolic biomarkers of disease and drug efficacy besides analyzing the global metabolic profile of the whole system. Comprehensive biochemical profiling using metabolomics has provided insights into the pathophysiological progression of diabetes not only in clinical but also in per-clinical conditions [15]. The metabolome, can be analyzed from different matrices such as serum, urine, cerebrospinal fluid, breath and tissues. Metabolomics studies have revealed significant elevation of certain amino acids and their derivatives in serum that strongly correlate with fasting hemoglobin $\mathrm{Alc}(\mathrm{HbAlc}) . \mathrm{HbA1C}$ is a clinically accepted form of hemoglobin that is measured primarily to identify the average plasma glucose concentration over prolonged periods of time [21]. Increased $\mathrm{HbA1C}$ values are strong indicators of developing diabetic complications [7]. Urine metabolome analysis across different species in diabetes have identified significant changes in nucleotide metabolism, including that of $\mathrm{N}$-methylnicotinamide and $\mathrm{N}$-methyl2-pyridone-5-carboxamide, which may provide unique biomarkers for following T2D mellitus progression $[17,22,23]$.

Thus, metabolomics provides a comprehensive snapshot of the disease process and helps investigator to assess the disease status of the subject under study and inform therapeutic decisions. Metabolomics applied to diabetes research, helps to obtain an overview of the disease onset and progression. Several therapeutic targets have been discovered through metabolomics $[12,24]$. Cross-species mapping of the lipid profile using metabolomics have helped investigators develop models to investigate early disease pathophysiology of diabetes $[25,26]$. The understanding of lipid profile at a tissue-specific level further facilitates understanding of the etiology of different complications associated with diabetes $[25,27]$

Comprehensive analytical studies in multi-factorial diseases as T2D have an edge over isolated knowledge of individual components. This approach offers an accurate mechanistic understanding of the complex disease phenotype since integrated behavior of a system is likely to be different from that of a single component [11,12]. Global biochemical studies began in the mid-twentieth century with the use of gas chromatography-mass spectrometry (GC-MS) and were accentuated by the availability of non-magnetic resonance (NMR) spectrometers $[28,29]$. Eventually Mass spectrometry MS or NMR evolved as analytical instruments of choice to detect metabolic changes for diagnostic purposes. Advancements in computational technologies helped integrate and annotate the endogenous metabolite data quantitated through one of the existing analytical platforms such as MS, NMR and chromatographic systems helping the researchers investigate the effect of integrated metabolism on human health [30].

The intrinsic diversity in chemical structure, size, abundance and reactivity of the pool of metabolites in any biological samples makes it challenging to identify and quantify all metabolites simultaneously in a single, highthroughput platform. Majority of the reported studies apply MS or NMR spectroscopy as the analytical instrument of choice [31-34] However, many other techniques including Fourier transform infrared and Raman spectroscopy have also been used [35-37]. Electrochemical detection for identification and quantification of electrochemically active metabolites from redox pathways has also been reported $[38,39]$. The use of different analytical platforms provides complimentary information that can be integrated for deeper metabolome coverage [40].

\section{Overview of Metabolomics Work Flow}

Typically, metabolomics studies are investigative in nature involving delineation of biomarkers of a disease $[6,19,41]$. These can further be subdivided as metabolic profiling, using an untargeted approach or metabolite identification and quantitation using a targeted approach. It is critical that the samples from each group under investigation are collected, stored and processed in standardized manner. A combination of analytical methods, ${ }^{1} \mathrm{H}$ NMR spectroscopy and LC-MS have been used to provide information on metabolic pathways known to be altered by insulin deficiency in diabetes $[11,15,26,32]$. In absence of a single common platform to identify and quantitate all metabolites in the same sample simultaneously, the comprehensive metabolic changes are assembled by consolidating data from different platforms. 
Citation: Preet A, Karve TM, Rizk N, Cheema AK (2012) Metabolomics: Approaches and Applications to Diabetes Research. J Diabetes Metab S6:001. doi:10.4172/2155-6156.S6-001

Raw data collected from different analytical platforms are preprocessed to remove inconsistencies resulting from instrument performance or sampling. QTOF Mass spectrometry is capable of resolving thousands of molecules in a single experiment and making accurate mass assignments. Greater sensitivity allows for the detection of low abundance metabolites missed by NMR $[42,43]$. The MS spectral data is further filtered and is de-convoluted. Standard publicly available tools like XCMS, Metalign, MZmine and MathDAMP help data preprocessing $[44,45]$. The conversion of continuous NMR data into a segmented version by bucketing or binning corrects data for peak shifts due to $\mathrm{pH}$ or ionic strength variations across different samples as well as reduces the data significantly simplifying the subsequent data analysis $[46,47]$. Another approach involves de-convolution of NMR spectra into individual components allowing identification and quantitation of individual components from a complex NMR spectrum [48].

The pre-processed metabolomics data can be further analyzed using supervised and unsupervised algorithms. Supervised methods include partial least squares discriminate analysis (PLS-DA), support vector machines (SVM) and discriminant function analysis (DFA) and univariate ANOVA and median fold change (MFC). Unsupervised methods include principal component analysis (PCA), supervised and self-organized maps. Schematic of a typical metabolomics study workflow is illustrated in Figure 2. These tools help the researcher delineate candidate markers that are significantly dysregulated in the experimental data set. A select panel of candidate markers is then further validated using independent cohorts or by repeating the study.

\section{Metabolomics for Diabetes Research}

Mass Spectrometry (MS) has been successfully employed to investigate different processes central to diabetes, such as nonenzymatic protein glycation where different hexose sugars would modify the proteins leading to their altered or diminished functions [49] or to obtain metabolic profiles in T2D patients [32,50-53] and for diabetes risk assessment [18]. Due to sensitivity and diverse chemical identification capabilities, MS is the tool of choice for obtaining broad metabolic profiles in conjunction with gas or liquid chromatography [54,55].

\section{Gas chromatography-mass spectroscopy (GC-MS)}

GC-MS is the oldest and a robust tool for qualitative metabolic profiling. GC-MS provides high chromatographic resolution and allows for non-targeted profiling for the discovery of novel metabolites and metabolic pathways [56,57]. GC-MS involves electron impact ionization wherein the GC column eluants are introduced into the source, ionized and fragmented to generate a characteristic fragmentation pattern and mass spectrum that is typically used for chemical identification. GC-MS has been extensively used as a discovery tool in steroid characterizations for clinical purposes $[56,58,59]$.

GC-MS has been used for the study of pathways of oxidative stress activated in diabetic macrovascular disease, both in primate and rodent models [60-62]. These studies emphasize the role of oxidized amino acids as potential markers for the assessment of oxidative damage. The chromatographic resolution capacity of the conventional GC has been further enhanced by a more recent technique known as Comprehensive GC X GC-MS that has been applied successfully in metabolomics [63,64]. This technique uses an additional column for two dimensional separation that significantly increases the analytical performance by improving the chromatography thereby expanding metabolome coverage $[65,66]$.

\section{Liquid chromatography-mass spectrometry (LC-MS)}

LC-MS involves interfacing of liquid chromatography platforms with mass spectrometers. LC provides metabolite separation by equilibration between a mobile liquid phase and a stationary solid (or liquid) phase. The coupling of liquid systems to mass spectrometry is facilitated by the use of electrospray as the commonly applied ionization technique. Application of LC-MS as a reliable technology has increased during the previous decade [18,67-69]. LC-MS metabolic profiling of 20 non-obese and obese individuals showed a strong correlation between fasting concentrations of branched-chain and aromatic amino acids and serum insulin [70]. A strong correlation has also been reported between branched-chain amino acid (BCAA) catabolism and insulin resistance [71]. LC-MS was used to generate metabolic profiles from 2,422 normo-glycemic individuals followed over a period of twelve years of which 201 eventually developed diabetes [18]. This study reported a panel of more than 60 metabolites including branched-chain and aromatic amino acids as predictors of development of diabetes over the standard risk factors such as fasting glucose, body mass index (BMI) etc.

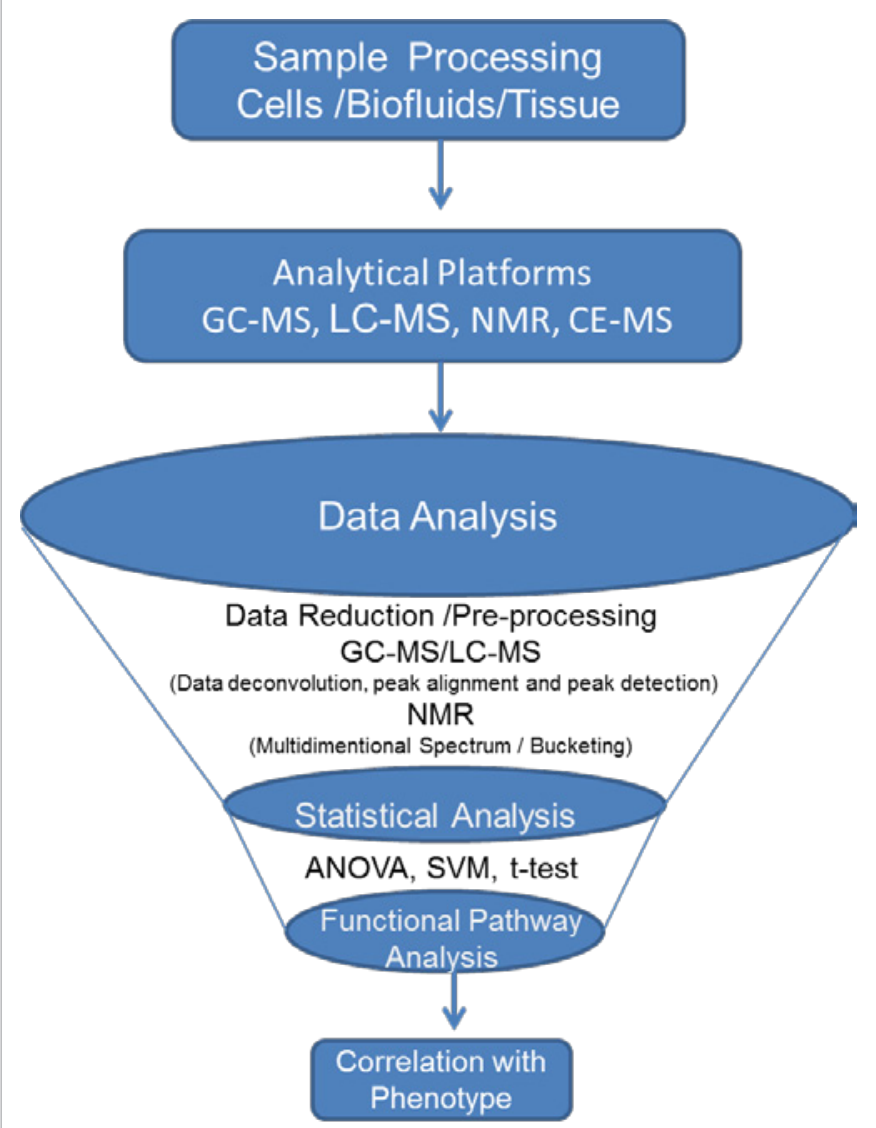

Figure 2: Schematic representation of a metabolomics experimental workflow Based on the study design, biological samples are collected and processed and subsequently analyzed using various analytical platforms. The different analytical platforms such as Gas chromatography-mass spectroscopy (GC-MS), Liquid chromatography-mass spectroscopy (LC-MS), Capillary Electrophoresismass spectroscopy (CE-MS) and Nuclear Magnetic Resonance Spectroscopy (NMR) are used for data acquisition. The raw data is pre-processed, reduced and subjected to statistical analysis using ANOVA, SVM, and/or student's t-test. The results help to establish a correlation with the phenotype and can also be utilized to confirm or generate hypothesis. 


\section{Capillary electrophoresis- mass spectrometry (CE-MS)}

Capillary Electrophoresis- Mass Spectrometry (CE-MS) is yet another analytical tool utilized for metabolite separation and detection. Metabolites are first separated by CE based on charge and size, and then selectively detected using MS by monitoring a large range of $\mathrm{m} / \mathrm{z}$ values. CE is particularly suited for the separation of polar and charged compounds and can provide complementary information to LC-MS on the biological composition of sample [72]. It has been successfully used in different studies to detect and quantitate cationic and anionic metabolites not only across different species but also across different sample types as bio-fluids, cells and tissues [73-78]. Cross-platform analysis utilizing CE-MS fingerprinting augmented the identification of metabolites galactosylhydroxylysine, l-carnitine, among others which markedly increased in urine from diabetic rats as compared to control animals [79].

\section{Nuclear magnetic resonance spectroscopy (NMR)}

NMR spectroscopy is a quantitative, highly reproducible and non-selective analytical technique for metabolic profiling [80]. It is independent of the hydrophobicity or $\mathrm{pKa}$ of the compounds being analyzed. It has been extensively used for metabolic profiling for more than 20 years. This technique interrogates all the molecules present in the sample simultaneously by using the active NMR of hydrogen $\left({ }^{1} \mathrm{H}\right)$ or carbon $\left({ }^{13} \mathrm{C}\right)$-the so called common magnetic nuclei [81]. The qualitative limitation of NMR lies in its inherent insensitivity and is hence suitable only for detection and quantification of metabolites present in relatively high concentration [12]. Alternative strategies are being currently developed to increase the sensitivity of NMR including the use of cryoprobes in improving signal to noise for ${ }^{13} \mathrm{C}$ NMR based metabolomics [82-84]. Another area of improvement is the use of hyperpolarized substrates to selectively enhance the resonance of key metabolites.

Low circulating levels of plasma phosphatidylcholine and high levels of methylamines were detected in plasma and urine samples from $129 S 6$ mice, a mouse strain known to be susceptible to hepatic steatosis and insulin resistance in comparison to BALBc (relative resistance) using ${ }^{1} \mathrm{H}$ NMR-based metabolic profiling [85]. In a recent study, assessment of biochemical process of diabetes has been done utilizing quantitative ${ }^{1} \mathrm{H}$ NMR-based metabonomics to analyze urine, serum, and liver extracts from streptozotocin-induced diabetic rats [86]. This study identified a number of metabolic alterations in liver samples from diabetic rats including metabolites participating in nitrogen and carbon metabolism. Salek et al used ${ }^{1} \mathrm{H}$ NMR to compare metabolic alterations not only in diabetic animal models but also in humans [22]. The study involved NMR based urine analysis from 12 healthy and 30 T2D patients. A clear group separation based on a large number of metabolites that included amino acids such as alanine, ornithine, etc. was observed. Although a robust technique, the detection limits of ${ }^{1} \mathrm{H}$ NMR is compromised by the large number of co-resonances that may be somewhat improvised by use of 2-dimensional NMR or with use of nuclei, such as ${ }^{13} \mathrm{C}$ that have more dispersion.

\section{Animal Models of Diabetes Mellitus}

Animal models, including but not limited to rabbits, dogs, monkeys and various murine species (e.g. rat and mice), serve a critical function in understanding of the pathophysiology, early embryonic clues of disease predisposition(s) and genetic basis of the disease as well as developing therapeutic (such as drug efficacy and toxicity) and/ or preventive strategies and tracking disease prognosis e.g. rhesus monkey models have been used to understand T1D as well as developing insulin administration strategies as a medical intervention to alleviate T1D [87]. Given the multi-faceted nature of diabetes mellitus, there are numerous murine models representing individual factors shown to be responsible for developing T1D or T2D. These murine models can be grouped as spontaneous or genetic models, diet or nutritional induced models, environmental or chemically induced models, surgically induced models and transgenic or knockout models $[88,89]$. Even though, none of the rodent models may accurately correlate with the human disease pathology for diabetes singularly, since most of these animals display an array of symptoms that resemble the human disease, they can be manipulated for studying the impact of a particular component such as genetics or environmental effect(s) $[89,90]$.

Spontaneous murine models, known since early 1980's, are available for both T1D and T2D, and are helpful in understanding the genetics of the disease, including consequences of inbreeding, insulin resistance mainly due to glucose toxicity, ketosis, obesity and hyperinsulinemia $[89,91]$. Some of the most commonly used murine models for genetic studies of diabetes are NOD (non-obese diabetic) mice, KK (Kuo Kondo) mice and BB (bio breeding) rats [88,91]. Mouse models such as NOD-Pdcd1-/- (programmed cell death 1 [PD-1, Pdcd1], an immuneinhibitory receptor from the CD28/cytotoxic T lymphocyte-associated antigen-4 family), have been further developed to specifically study T1D [92]. A recent study illustrating urine profile in a spontaneous non-human primate T2D model, Rhesus monkey (Rhesus macaques), was able to detect a defective $\mathrm{Na}(+)$-dependent transporter, SLC6A20, in proximal tubules of kidneys [93]. Further, this study confirmed similar observation in $\mathrm{db} / \mathrm{db}$ mouse model and thus reflects the basic functional changes at the cellular level in a disease state of T2DM.

Diet or nutritionally-induced models play an important role in gaining insights specifically concerning T2D, which is mainly linked with the obesity and development of insulin resistance leading to glucose toxicity. As per the information by the American Diabetic Association, T2D is much more common in ethnic minorities as well as other non-white communities such as Asian Americans in the United States and is linked mainly to the diet. A diet high in saturated fats and poor in nutritional value is a major contributing element to obesity and in turn developing T2D. Number of studies using animal models have highlighted the role of factors apart from high-fat diet that may influence the predisposition of these populations for developing T2D; source of protein in diet [94]. In these studies, rats that were on diets high in fats and had most of their protein share from casein-based food or soy products showed insulin resistance while the group of animals with high fat diet and most of its proteins coming from cod (fish-based) showed no insulin resistance [94]. Thus, these studies suggest that the overall food pyramid distribution and sources of each particular food groups influence the outcome, at least, in predicting the predisposition of a population for developing T2D.

Another subtype of murine model, environmental or chemicalinduced rodent model, has been useful in various studies focused on disorders related to diabetes. One of the most commonly used chemicalinduced diabetic murine models is streptozotocin (STZ)-induced diabetic rat $[88,95]$. Diabetes, a heterogeneous disease, is known to affect a number of normal functions in various human organs. One of the common disorders observed in diabetic men is diabetes-related erectile dysfunction (ED), which has been widely studied in chemicalinduced murine models [95,96]. Maggi et al reported that STZ-diabetic rats suffered from the hypogonadism, a condition frequently observed in diabetics, along with low testosterone production and atrophy in a 
number of androgen-dependent accessory glands as well as induction of RhoA/Rho-kinase (ROCK) signaling pathway [95,96]. Further, the authors found that normalizing the testosterone production along with the introduction of ROCK inhibitors showed significant improvement in alleviating the ED in these animals and may thus have a promising potential for clinical implications for the patients suffering from diabetes-related ED.

A different type of animal model, a surgically-induced model in mice, pigs, dogs and rats, has been helpful in studying diabetic-related retinopathy and the role of pancreas in T1D and T2D [97]. The surgical models are generally developed by complete or partial removal of pancreas known as pancreatectomy, $\mathrm{He}$, et al. have shown that complete pancreatectomy is one of the two factors in diabetic monkeys that can lead to severe hypoglycemic conditions [98]. Further, combining the pancreatectomy along with the other diabetic murine models such as spontaneous rat model has led to some interesting findings. Plachot, et al. found that even partial pancreatectomy in GK (Goto-Kakizaki) rats, a commonly used spontaneous rat model to study T2D further accelerates the disease initiation by reducing beta-cell proliferation and insulin secretion, a critical element in diabetes [99]. Hyperglycemia, responsible for the loss or reduction of beta-cell proliferation has also been connected to the expression of $c-m y c$, a known oncogene, and other transcription factors in these murine models and thus, may indirectly control the insulin production in these animals [100].

Transgenic or knockout murine models are other common tools that are used to study the role of specific gene(s) associated with a disease such as neurodegenerative disorder, cancer and many other genetic diseases. Most of the transgenic rodent models for diabetes, $\mathrm{T} 1 \mathrm{D}$ and $\mathrm{T} 2 \mathrm{D}$, are associated with single or double knockout of various genes in the insulin production pathway [101]. However, due to number of components in a insulin synthesis pathway and resultant insulin resistance in diabetes, transgenic models have been disappointing to this end [101]. Nevertheless, transgenic or knockout models are useful when used in conjunction with targeted molecular and biochemical studies. Transgenic mouse models are also helpful in studying diabetes in conjunction with other obesity-related metabolic disorders/ syndromes such as atherosclerosis, dyslipidemia and insulin resistance and thus are frequently used in metabolomics studies, specifically LC-MS-based lipidomics and liver and blood profiling in these animal models $[90,102,103]$. At least one MS-based lipidomics study found that the stimulation of PPAR $\gamma$ (peroxisome proliferation activated receptors) by an agonist rosiglitazone as detected in blood plasma of obese T2D mouse model and thus has a promising potential as a target for drug therapy in T2D [102,104].

\section{Future Directions/ Challenges}

Given the recently released predictions about escalating prevalence of diabetes worldwide by the WHO, it is extremely critical to integrate biochemical, molecular and clinical knowledge to design diabetes treatment and preventive strategies that are affordable as well as effective. The modern improvements in technology such as advances in the multi-platform and highthroughput omics; transcriptomics, proteomics and metabolomics, and vast bioinformatics toolset, as well as advanced knowledge acquired by molecular and biochemical studies are certainly helpful. However, the immense data obtained from the "omics" need to be interpreted with a great caution; giving due consideration to the limitations of animal models and the statistical tools used. Results should thus be interpreted in conjunction with experimental evidence from biochemical and molecular studies.
Currently metabolomics approaches lack standardized procedures for sample preparation, data analysis and interpretation. Thus, there is a critical need to develop common benchmarks describing the experimental set-up and ontology for metabolomics similar to the universal standards available for other "omics" such as transcriptomics e.g. MIAME (minimum information associated with a microarray experiment) [105] and proteomics e.g. MIAPE (minimum information about a proteomics experiment) [106]. Additionally, there is a lack of comprehensively annotated metabolite databases for unambiguous metabolite identification [107].

Similar caution is required in interpreting data obtained from the animal models since most animal models do not entirely complement various disease states as found in T1D and T2D in humans. The use of non-human primates closest to humans such as monkeys can be successfully substituted for a better understanding of T1D and T2D. However, the usage of primates requires complex animal care protocols and is not cost-effective in a large experimental set-up. Additionally, since they have a longer life span the interpretation of results can be delayed and complex.

Nonetheless, the data obtained from integrating various "omics" studies, may be useful in discovering molecular markers and/ or drug targets that can be of clinical significance for developing therapeutic interventions as well as for gaining mechanistic insights into disease onset and progression. In addition, these and other studies may also have implications in unraveling genetic markers predicting individual's predisposition for developing diabetes and thus can be valuable in formulating diagnostic tests for early detection of diabetes.

\section{Acknowledgements}

Drs. Cheema and Rizk are also supported in part, by the Qatar National Research foundation grant (NPRP 08-740-3-148).

\section{References}

1. Preet A, Gupta BL, Siddiqui MR, Yadava PK, Baquer NZ (2005) Restoration of ultrastructural and biochemical changes in alloxan-induced diabetic rat sciatic nerve on treatment with Na3VO4 and Trigonella-a promising antidiabetic agent Mol Cell Biochem 278: 21-31.

2. Kahn C (1994) Banting lecture: Insulin action, diabetogenes, and the cause of type II diabetes. Diabetes 43: 1066-1084.

3. Wild S, Roglic G, Green A, Sicree R, King H (2004) Global Prevalence of Diabetes. Diabetes Care 27: 1047-1053.

4. Cowie CC, Rust KF, Byrd-Holt DD, Eberhardt MS, Flegal KM, et al. (2006) Prevalence of Diabetes and Impaired Fasting Glucose in Adults in the U.S. Population. Diabetes Care 29: 1263-1268.

5. Expert Committee on the Diagnosis and Classification of Diabetes Mellitus (2003) Report of the Expert Committee on the Diagnosis and Classification of Diabetes Mellitus. Diabetes Care 26: S5-S20.

6. Chen L, Magliano DJ, Zimmet PZ (2011) The worldwide epidemiology of type 2 diabetes mellitus[mdash]present and future perspectives. Nat Rev Endocrino doi: 10.1038/nrendo.2011.183.

7. Knuiman MW, Welborn TA, McCann VJ, Stanton KG, Constable IJ (1986) Prevalence of diabetic complications in relation to risk factors. Diabetes 35 1332-1339.

8. Goehring I, Sauter NS, Catchpole G, Assmann A, Shu L, et al. (2011) Identification of an intracellular metabolic signature impairing beta cell function in the rat beta cell line INS-1E and human islets. Diabetologia 54: 2584-2594.

9. Pflueger $M$, Seppänen-Laakso $T$, Suortti $T$, Hyötyläinen $T$, Achenbach $P$, et al. (2011) Age- and Islet Autoimmunity-Associated Differences in Amino Acid and Lipid Metabolites in Children at Risk for Type 1 Diabetes. Diabetes 60 2740-2747.

10. Ha CY, Kim JY, Paik JK, Kim OY, Paik YH, et al. (2011) The association of specific metabolites of lipid metabolism with markers of oxidative stress, 
Citation: Preet A, Karve TM, Rizk N, Cheema AK (2012) Metabolomics: Approaches and Applications to Diabetes Research. J Diabetes Metab S6:001. doi:10.4172/2155-6156.S6-001

inflammation and arterial stiffness in men with newly diagnosed type 2 Diabetes. Clin Endocrinol: 1365-2265.

11. Dunn WB, Goodacre R, Neyses L, Mamas M (2011) Integration of metabolomics in heart disease and diabetes research: current achievements and future outlook. Bioanalysis 3: 2205-2222.

12. Dunn WB, Broadhurst DI, Atherton HJ, Goodacre R, Griffin JL (2011) Systems level studies of mammalian metabolomes: the roles of mass spectrometry and nuclear magnetic resonance spectroscopy. Chemical Society Reviews 40: 387426

13. Ma H, Sorokin A, Mazein A, Selkov A, Selkov E, et al. (2007) The Edinburgh human metabolic network reconstruction and its functional analysis. Mol Syst Biol 3: 1-8

14. Duarte NC, Becker SA, Jamshidi N, Thiele I, Mo ML, et al. (2007) Globa reconstruction of the human metabolic network based on genomic and bibliomic data. Proc Natl Acad of Sci USA104: 1777-1782.

15. Suhre K, Meisinger C, Döring A, Altmaier E, Belcredi P, et al. (2010) Metabolic Footprint of Diabetes: A Multiplatform Metabolomics Study in an Epidemiological Setting. PLoS ONE 5: e13953.

16. Altmaier E, Ramsay SL, Graber A, Mewes HW, Weinberger KM, et al. (2008) Bioinformatics Analysis of Targeted Metabolomics-Uncovering Old and New Tales of Diabetic Mice under Medication. Endocrinology 149: 3478-3489.

17. Griffin J, Atherton HJ, Steinbeck C, Salek RM (2011) A Metadata description of the data in "A metabolomic comparison of urinary changes in type 2 diabetes in mouse, rat, and human.". BMC Research Notes 4: 272.

18. Wang TJ, Larson MG, Vasan RS, Cheng S, Rhee EP, et al. (2011) Metabolite profiles and the risk of developing diabetes. Nat Med 17: 448-453.

19. Kus V, Flachs P, Kuda O, Bardova K, Janovska P, et al. (2011) Unmasking Differential Effects of Rosiglitazone and Pioglitazone in the Combination Treatment with $n-3$ Fatty Acids in Mice Fed a High-Fat Diet. PLoS ONE 6 e27126.

20. Horgan RP, Broadhurst DI, Walsh SK, Dunn WB, Brown M, et al. (2011) Metabolic Profiling Uncovers a Phenotypic Signature of Small for Gestational Age in Early Pregnancy. J Proteome Res 10: 3660-3673.

21. Fiehn O, Garvey WT, Newman JW, Lok KH, Hoppel CL, et al. (2010) Plasma Metabolomic Profiles Reflective of Glucose Homeostasis in Non-Diabetic and Type 2 Diabetic Obese African-American Women. PLoS ONE 5: e15234.

22. Salek RM, Maguire ML, Bentley E, Rubtsov DV, Hough T, et al. (2007) A metabolomic comparison of urinary changes in type 2 diabetes in mouse, rat, and human. Physiol Genomics 29: 99-108.

23. Zhao L, Gao H, Lian F, Liu X, Zhao Y, et al. (2011) 1H-NMR-based metabonomic analysis of metabolic profiling in diabetic nephropathy rats induced by streptozotocin. Am J Physiol Renal Physiol 300: F947-F956.

24. Yuan W, Zhang J, Li S, Edwards JL (2011) Amine Metabolomics of Hyperglycemic Endothelial Cells using Capillary LC-MS with Isobaric Tagging. J Proteome Res 10: 5242-5250.

25. Sysi-Aho M, Ermolov A, Gopalacharyulu PV, Tripathi A, Seppänen-Laakso T, et al. (2011) Metabolic Regulation in Progression to Autoimmune Diabetes. PLoS Comput Biol 7: e1002257.

26. Griffin JL, Nicholls AW (2006) Metabolomics as a functional genomic tool for understanding lipid dysfunction in diabetes, obesity and related disorders. Pharmacogenomics 7: 1095-1107.

27. Macotela Y, Emanuelli B, Bång AM, Espinoza DO, Boucher J, et al. (2011) Dietary Leucine - An Environmental Modifier of Insulin Resistance Acting on Multiple Levels of Metabolism. PLoS ONE 6: e21187.

28. Behar KL, den Hollander JA, Stromski ME, Ogino T, Shulman RG, et al. (1983) High-resolution $1 \mathrm{H}$ nuclear magnetic resonance study of cerebral hypoxia in vivo. Proc Natl Acad Sci USA 80: 4945-4948.

29. Linus Pauling, Robinson AB, Teranishi R, Cary P (1971) Quantitative Analysis of Urine Vapor and Breath by Gas-Liquid Partition Chromatography. Proc Nat Acad Sci U S A 68: 2374-2376.

30. Zivkovic AM, JB German (2009) Metabolomics for assessment of nutritional status. Curr Opin Clin Nutr Metab Care 12: 501-507.

31. Rhee EP, Gerszten RE (2012) Metabolomics and Cardiovascular Biomarker Discovery. Clin Chem 58: 137-149.
32. Lanza IR, Zhang S, Ward LE, Karakelides H, Raftery D, et al. (2010) Quantitative Metabolomics by H-NMR and LC-MS/MS Confirms Altered Metabolic Pathways in Diabetes. PLoS ONE 5: e10538.

33. Ruiz-Aracama A, Peijnenburg A, Kleinjans J, Jennen D, van Delft J, et al. (2011) An untargeted multi-technique metabolomics approach to studying intracellular metabolites of HepG2 cells exposed to 2,3,7,8-tetrachlorodibenzop-dioxin. BMC Genomics 12: 251

34. Napoli C, Sperandio N, Lawlor RT, Scarpa A, Molinari H, et al. (2011) Urine Metabolic Signature of Pancreatic Ductal Adenocarcinoma by $1 \mathrm{H}$ Nuclear Magnetic Resonance: Identification, Mapping, and Evolution. J Proteome Res: p. in press.

35. Wu H, Volponi JV, Oliver AE, Parikh AN, Simmons BA, et al. (2011) In vivo lipidomics using single-cell Raman spectroscopy. Proc Natl Acad Sci 108 3809-3814.

36. Krishna CM, Kurien J, Mathew S, Rao L, Maheedhar K, et al. (2008) Raman spectroscopy of breast tissues. Expert Rev Mol Diagn 8: 149-166.

37. Guo K, Peng J, Zhou R, Li L (2011) Ion-pairing reversed-phase liquid chromatography fractionation in combination with isotope labeling reversedphase liquid chromatography-mass spectrometry for comprehensive metabolome profiling. J Chromatogr A 1218: 3689-3694.

38. Chen SP, Wu J, Yu XD, Xu JJ, Chen HY (2010) Multi-parameter detection of diabetes mellitus on multichannel poly(dimethylsiloxane) analytical chips coupled with nanoband microelectrode arrays. Electrophoresis 31: 3097-3106.

39. Hrydziuszko O, Silva MA, Perera MT, Richards DA, Murphy N, et al. (2010) Application of Metabolomics to Investigate the Process of Human Orthotopic Liver Transplantation: A Proof-of-Principle Study. OMICS 14: 143-150.

40. Zhang A, Sun H, Wang P, Han Y, Wang X (2012) Modern analytical techniques in metabolomics analysis. Analyst 137: 293-300.

41. Connor SC, Hansen MK, Corner A, Smith RF, Ryan TE (2010) Integration of metabolomics and transcriptomics data to aid biomarker discovery in type 2 diabetes. Mol BioSyst 6: 909-921.

42. Connor SC, Wu W, Sweatman BC, Manini J, Haselden JN, et al. (2004) Effects of feeding and body weight loss on the $1 \mathrm{H}$-NMR-based urine metabolic profiles of male Wistar Han rats: implications for biomarker discovery. Biomarkers 9 : p. $156-79$.

43. Griffin JL, Williams HJ, Sang E, Nicholson JK (2001) Abnormal lipid profile of dystrophic cardiac tissue as demonstrated by one- and two-dimensional magicangle spinning 1H NMR spectroscopy. Magn Reson Med 46: 249-255.

44. Katajamaa M, Miettinen J, Orešič M (2006) MZmine: toolbox for processing and visualization of mass spectrometry based molecular profile data. Bioinformatics 22: 634-636.

45. Baran R, Kochi H, Saito N, Suematsu M, Soga T, et al. (2006) MathDAMP: a package for differential analysis of metabolite profiles. BMC Bioinformatics 7 : 530 .

46. Goodacre R, Vaidyanathan S, Dunn WB, Harrigan GG, Kell DB (2004) Metabolomics by numbers: acquiring and understanding global metabolite data. Trends Biotechnol 22: 245-252.

47. Kuhl C, Tautenhahn R, Böttcher C, Larson TR, Neumann S (2011) CAMERA An Integrated Strategy for Compound Spectra Extraction and Annotation of Liquid Chromatography/Mass Spectrometry Data Sets. Anal Chem.

48. Schripsema J (2010) Application of NMR in plant metabolomics: techniques, problems and prospects. Phytochem Anal 21: 14-21.

49. Lapolla A, Fedele D, Traldi P (2011) Diabetes and mass spectrometry. Diabetes Metab Res Rev 17: 99-112.

50. Wang C, Kong H, Guan Y, Yang J, Gu J, et al. (2005) Plasma phospholipid metabolic profiling and biomarkers of type 2 diabetes mellitus based on highperformance liquid chromatography/electrospray mass spectrometry and multivariate statistical analysis. Anal Chem 77: 4108- 4116.

51. Xia JF, Liang QL, Liang XP, Wang YM, Hu P, et al. (2009) Ultraviolet and tandem mass spectrometry for simultaneous quantification of 21 pivotal metabolites in plasma from patients with diabetic nephropathy. $\mathrm{J}$ Chromatogr $\mathrm{B}$ Analyt Technol Biomed Life Sci 877: 1930-1936.

52. Zhang J, Yan L, Chen W, Lin L, Song X, et al. (2009) Metabonomics research of diabetic nephropathy and type 2 diabetes mellitus based on UPLC-oaTOFMS system. Anal Chim Acta 650: 16-22. 
Citation: Preet A, Karve TM, Rizk N, Cheema AK (2012) Metabolomics: Approaches and Applications to Diabetes Research. J Diabetes Metab S6:001. doi:10.4172/2155-6156.S6-001

53. Verrijn Stuart AA, Schipper HS, Tasdelen I, Egan DA, Prakken BJ, et al. (2011) Altered Plasma Adipokine Levels and in Vitro Adipocyte Differentiation in Pediatric Type 1 Diabetes. J Clin Endocrinol Metab.

54. Dettmer K, Aronov PA, Hammock BD (2007) Mass spectrometry-based metabolomics. Mass Spectrom Rev 26: 51-78.

55. Dunn WB, Broadhurst D, Brown M, Baker PN, Redman CW, et al (2008) Metabolic profiling of serum using Ultra Performance Liquid Chromatography and the LTQ-Orbitrap mass spectrometry system. J Chromatogr B Analyt Technol Biomed Life Sci 871: 288-298.

56. Krone N, Hughes BA, Lavery GG, Stewart PM, Arlt W, et al. (2010) Gas chromatography/mass spectrometry (GC/MS) remains a pre-eminent discovery tool in clinical steroid investigations even in the era of fast liquid chromatography tandem mass spectrometry (LC/MS/MS). J Steroid Biochem Mol Biol 121: 496504

57. Ng DP, Salim A, Liu Y, Zou L, Xu FG, et al. (2011) A metabolomic study of low estimated GFR in non-proteinuric type 2 diabetes mellitus. Diabetologia: 55: 499-508.

58. Arlt W, Biehl M, Taylor AE, Hahner S, Libé R, et al. (2011) Urine Steroid Metabolomics as a Biomarker tool for detecting malignancy in adrenal tumors. J Clin Endocrinol Metab 96: 3775-3784.

59. Menéndez-Carreño $M$, Varo N, Mugueta $C$, Restituto $P$, Ansorena $D$, et al (2011) Correlation between serum content of the main COPs (cholesterol oxidation products) from autoxidation and cardiovascular risk factors. Nutr Hosp 26: 144-151.

60. Vivekanadan-Giri A, Wang JH, Byun J, Pennathur S (2008) Mass spectrometric quantification of amino acid oxidation products identifies oxidative mechanisms of diabetic end-organ damage. Rev Endocr Metab Disord 9: 275-287.

61. Tavafi M, Ahmadvand H, Tamjidipoor A, Delfan B, Khalatbari AR (2011) Satureja khozestanica essential oil ameliorates progression of diabetic nephropathy in uninephrectomized diabetic rats. Tissue Cell 43: 45-51.

62. Fan X, Sell DR, Zhang J, Nemet I, Theves M, et al. (2010) Anaerobic vs aerobic pathways of carbonyl and oxidant stress in human lens and skin during aging and in diabetes: A comparative analysis. Free Radic Biol Med 49: 847-856.

63. Koek MM, Muilwijk B, van Stee LL, Hankemeier T (2008) Higher mass loadability in comprehensive two-dimensional gas chromatography-mass spectrometry for improved analytical performance in metabolomics analysis. J Chromatogr A 1186: 420-429.

64. Pierce KM, Hoggard JC, Mohler RE, Synovec RE (2008) Recent advancements in comprehensive two-dimensional separations with chemometrics. J Chromatogr A 1184: 341-352

65. John Heim (2010) Analyzing Small Molecule Metabolite Profiles of Diabetic and Nondiabetic Urine Samples Using GCXGC-TOF-MS and Statistical Software as a Data-Mining Strategy.

66. Huang X, Regnier FE (2007) Differential metabolomics using stable isotope labeling and two-dimensional gas chromatography with time-of-flight mass spectrometry. Anal Chem 80: 107-114.

67. Allwood JW, Goodacre R (2010) An introduction to liquid chromatographymass spectrometry instrumentation applied in plant metabolomic analyses. Phytochem Anal 21: 33-47.

68. Becker S, Kortz L, Helmschrodt C, Thiery J, Ceglarek U (2011) LC-MS-based metabolomics in the clinical laboratory. J Chromatogr B Analyt Technol Biomed Life Sci Oct 22 in press.

69. Tsutsui H, Maeda T, Toyo'oka T, Min JZ, Inagaki S, et al. (2010) Practical analytical approach for the identification of biomarker candidates in prediabetic state based upon metabonomic study by ultraperformance liquid chromatography coupled to electrospray ionization time-of-flight mass spectrometry. J Proteome Res 9: 3912-3922.

70. Felig P, Marliss E, Cahill GF (1969) Plasma amino acid levels and insulin secretion in obesity. N Engl J Med 281: 811-816.

71. Newgard CB, An J, Bain JR, Muehlbauer MJ, Stevens RD, et al. (2009) A branched-chain amino acid-related metabolic signature that differentiates obese and lean humans and contributes to insulin resistance. Cell Metab 9: 311-326.

72. Ramautar R, Somsen GW, de Jong GJ (2009) CE-MS in metabolomics. Electrophoresis 30: 276-291.
73. Allard E, Bäckström D, Danielsson R, Sjöberg PJ, Bergquist J (2008) Comparing capillary electrophoresis-mass spectrometry fingerprints of urine samples obtained after intake of coffee, tea, or water. Anal Chem 80: 89468955.

74. Sugimoto M, Wong DT, Hirayama A, Soga T, Tomita M (2010) Capillary electrophoresis mass spectrometry-based saliva metabolomics identified oral, breast and pancreatic cancer-specific profiles. Metabolomics 6: 78-95.

75. Lee R, West D, Phillips SM, Britz-McKibbin P (2010) Differential metabolomics for quantitative assessment of oxidative stress with strenuous exercise and nutritional intervention: thiol-specific regulation of cellular metabolism with $\mathrm{N}$-acetyl-L-cysteine pretreatment. Anal Chem 82: 2959-2968.

76. Kato Y, Kubo Y, Iwata D, Kato S, Sudo T, et al. (2010) Gene knockout and metabolome analysis of carnitine/organic cation transporter OCTN1. Pharm Res 27: 832-840.

77. Sakuragawa T, Hishiki T, Ueno Y, Ikeda S, Soga T, et al. (2010) Hypotaurine is an Energy-Saving Hepatoprotective Compound against Ischemia-Reperfusion Injury of the Rat Liver. J Clin Biochem Nutr 46: 126-134.

78. Ramautar R, Toraño JS, Somsen GW, de Jong GJ (2010) Evaluation of CE methods for global metabolic profiling of urine. Electrophoresis 31: 2319-2327.

79. Moraes EP, Rupérez FJ, Plaza M, Herrero M, Barbas C (2011) Metabolomic assessment with CE-MS of the nutraceutical effect of Cystoseira spp extracts in an animal model. Electrophoresis 32: 2055-2062.

80. Bothwell JH, Griffin JL (2011) An introduction to biological nuclear magnetic resonance spectroscopy. Biol Rev Camb Philos Soc 86: 493-510.

81. Nicholson JK, Wilson ID (2003) Opinion: understanding 'global' systems biology: metabonomics and the continuum of metabolism. Nat Rev Drug Discov 2: 668-676.

82. Keun HC, Beckonert O, Griffin JL, Richter C, Moskau D, et al. (2002) Cryogenic probe 13C NMR spectroscopy of urine for metabonomic studies. Anal Chem 74: $4588-4593$

83. Reinhard Arnold M, Robert Kalbitzer H, Kremer W (2003) High-sensitivity sapphire cells for high pressure NMR spectroscopy on proteins. J Magn Reson 161: 127-131.

84. Lane AN, Arumugam S (2005) Improving NMR sensitivity in room temperature and cooled probes with dipolar ions. J Magn Reson 173: 339-343.

85. Dumas ME, Barton RH, Toye A, Cloarec O, Blancher C, et al. Metabolic profiling reveals a contribution of gut microbiota to fatty liver phenotype in insulin-resistant mice. Proc Natl Acad Sci U S A 103: 12511-12516.

86. Zhao L, Liu X, Xie L, Gao H, Lin D (2010) 1H NMR-based Metabonomic Analysis of Metabolic Changes in Streptozotocin-induced Diabetic Rats. Anal Sci 26: 1277-1282.

87. Qiao CF, Tian BL, Mai G, Wei LL, Jin X, et al. (2009) Induction of diabetes in rhesus monkeys and establishment of insulin administration strategy. Transplant Proc 41: 413-417.

88. Srinivasan K, Ramarao P (2007) Animal models in type 2 diabetes research: an overview. Indian J Med Res 125: 451-472.

89. Mordes JP, Rossini AA (1981) Animal models of diabetes. Am J Med 70: 353360

90. van Ginneken V, Verhey E, Poelmann R, Ramakers R, van Dijk KW, et al (2007) Metabolomics (liver and blood profiling) in a mouse model in response to fasting: a study of hepatic steatosis. Biochim Biophys Acta 1771: 1263-1270.

91. Rees DA, Alcolado JC (2005) Animal models of diabetes mellitus. Diabet Med 22: $359-370$

92. Wang J, Yoshida T, Nakaki F, Hiai H, Okazaki T, et al. (2005) Establishment of NOD-Pdcd1-/- mice as an efficient animal model of type I diabetes. Proc Nat Acad Sci U S A 102: 11823-11828.

93. Patterson AD, Bonzo JA, Li F, Krausz KW, Eichler GS, et al. (2011) Metabolomics reveals attenuation of the SLC6A20 kidney transporter in nonhuman primate and mouse models of type 2 diabetes mellitus. J Biol Chem 286: 19511-19522.

94. Storlien LH, Higgins JA, Thomas TC, Brown MA, Wang HQ, et al. (2000) Die composition and insulin action in animal models. $\mathrm{Br} J$ Nutr 83: S85-90. 
Citation: Preet A, Karve TM, Rizk N, Cheema AK (2012) Metabolomics: Approaches and Applications to Diabetes Research. J Diabetes Metab S6:001. doi:10.4172/2155-6156.S6-001

Page 8 of 8

95. Zhang XH, Filippi S, Morelli A, Vignozzi L, Luconi M, et al. (2006) Testosterone restores diabetes-induced erectile dysfunction and sildenafil responsiveness in two distinct animal models of chemical diabetes. J Sex Med 3: 253-264.

96. Vignozzi L, Morelli A, Filippi S, Ambrosini S, Mancina R, et al. (2007) Testosterone regulates RhoA/Rho-kinase signaling in two distinct animal models of chemical diabetes. J Sex Med 4: 620-630.

97. Engerman RL, Kern TS (1995) Retinopathy in animal models of diabetes. Diabetes Metab Rev 11: 109-120.

98. He S, Chen Y, Wei L, Jin X, Zeng L, et al. (2011) Treatment and risk factor analysis of hypoglycemia in diabetic rhesus monkeys. Exp Biol Med 236: 212 218

99. Plachot C, Movassat J, Portha B (2001) Impaired beta-cell regeneration after partial pancreatectomy in the adult Goto-Kakizaki rat, a spontaneous model of type II diabetes. Histochem Cell Biol 116: 131-139.

100. Jonas JC, Sharma A, Hasenkamp W, Ilkova H, Patanè G, et al. (1999) Chronic hyperglycemia triggers loss of pancreatic beta cell differentiation in an animal model of diabetes. J Biol Chem 274: 14112-14121.

101.Roep BO, Atkinson M, von Herrath M (2004) Satisfaction (not) guaranteed: re-evaluating the use of animal models of type 1 diabetes. Nat Rev Immunol 4: 989-997.

102. Watkins SM, Reifsnyder PR, Pan HJ, German JB, Leiter EH (2002) Lipid metabolome-wide effects of the PPARgamma agonist rosiglitazone. J Lipid Res 43: 1809-1817.

103. Griffin JL (2006) Understanding mouse models of disease through metabolomics. Curr Opin Chem Biol 10: 309-315.

104. Roberts LD, Murray AJ, Menassa D, Ashmore T, Nicholls AW, et al. (2011) The contrasting roles of PPARdelta and PPARgamma in regulating the metabolic switch between oxidation and storage of fats in white adipose tissue. Genome Biol 12: R75.

105. Brazma A (2009) Minimum Information About a Microarray Experiment (MIAME)--successes, failures, challenges. ScientificWorldJournal 9: 420-423.

106. Taylor CF, Paton NW, Lilley KS, Binz PA, Julian RK, et al. (2007) The minimum information about a proteomics experiment (MIAPE). Nat Biotechno 25: 887-893.

107. Griffin JL, Atherton HJ, Steinbeck C, Salek RM (2011) A Metadata description of the data in "A metabolomic comparison of urinary changes in type 2 diabetes in mouse, rat, and human." BMC Res Notes 4: 272
This article was originally published in a special issue, Metabolomics: Diabetics handled by Editor(s). Dr. Ippei Kanazawa, Shimane University Faculty of Medicine, Japan 